\title{
Robotic Astronomy with the Faulkes Telescopes and Las Cumbres Observatory Global Telescope
}

\author{
Fraser Lewis, ${ }^{1,2,3}$ Rachel Street, ${ }^{3}$ Paul Roche, ${ }^{1,2,3}$ Vanessa Stroud, ${ }^{1,2,3}$ and David M. Russell ${ }^{4}$ \\ ${ }^{1}$ Faulkes Telescope Project, School of Physics and Astronomy, 5, The Parade, Cardiff CF24 3AA, UK \\ ${ }^{2}$ Department of Physics and Astronomy, The Open University, Milton Keynes MK7 6AA, UK \\ ${ }^{3}$ Las Cumbres Observatory Global Telescope, 6740 Cortona Drive, Suite 102, Goleta, CA 93117, USA \\ ${ }^{4}$ Astronomical Institute "Anton Pannekoek", University of Amsterdam, P. O. Box 94249, 1090 GE, Amsterdam, The Netherlands \\ Correspondence should be addressed to Fraser Lewis, lewisf@cf.ac.uk
}

Received 30 June 2009; Accepted 2 December 2009

Academic Editor: Alberto J. Castro-Tirado

Copyright ( $) 2010$ Fraser Lewis et al. This is an open access article distributed under the Creative Commons Attribution License, which permits unrestricted use, distribution, and reproduction in any medium, provided the original work is properly cited.

We present results from ongoing science projects conducted by members of the Faulkes Telescope (FT) team and Las Cumbres Observatory Global Telescope (LCOGT). Many of these projects incorporate observations carried out and analysed by FT users, comprising amateur astronomers and schools. We also discuss plans for the further development of the LCOGT network.

\section{Introduction}

The Faulkes Telescope Project (http://www.faulkes-telescope .com/)is based in Cardiff, Wales, and is an educational and research arm of LCOGT (http://lcogt.net/), based in Goleta, California. Presently, the network has two operational 2-metre optical telescopes, located at Haleakala on Maui, Hawaii (FT North) and Siding Spring in New South Wales, Australia (FT South).

The FT provides telescope time free of charge to all UK schools, as well as other educational groups, and is able to provide its users with science and imaging projects [1]. Many of these projects relate to the UK school's curriculum, and some of them allow the schools to collaborate with researchers within the FT Team, and externally.

\section{The Telescopes}

Both FT North and FT South are 2-metre, fully autonomous, robotic Cassegrain-type reflectors with Richey-Chretien hyperbolic optics on an alt-azimuth mount. Both telescopes employ a Robotic Control System (RCS) [2].

They are currently operated in the following two modes.

(i) UK (and some overseas) schools use a "real-time interface" (RTI) mode, where each user has exclusive control of the telescope for 30 minutes at a time. During this time, the users are able to select co-ordinates, filters, and exposure times. Many educational users participate in data gathering for research programmes.

(ii) The "offline" mode of the telescope lends itself more easily to science observing, using a Graphic User Interface (GUI). In this instance, users may specify "flexible" (observe when most suitable), "fixed-time" (for specifically timed transient events), or "monitor" (repeat these observations every few days/weeks) type observations. For either mode, the telescopes also have an over-ride capability for Target of Opportunity ( $\mathrm{ToO}$ ) events such as gamma-ray bursts.

All observations are pipeline-processed to take account of flat-fielding and debiasing, with the finished images in $2 \times$ 2 binned FITS format available typically 24 hours after the observing run.

The telescopes are equipped with a $4.6^{\prime} \times 4.6^{\prime}$ field of view Merope camera [3] featuring SDSS $u^{\prime} g^{\prime} r^{\prime} i^{\prime} z^{\prime}$, Bessel BVRI, Pan-STARRS Z,Y broad-band filters in addition to $\mathrm{H} \alpha$, $\mathrm{H} \beta$ and $\mathrm{O}$ III narrow-band filters. The cameras use an E2V CCD42-40DD CCD with $2048 \times 2048$ pixels.

A second camera has recently been installed, known as the Spectral camera, possessing a wider field of view of $10.5^{\prime} \times 10.5^{\prime}$. The Spectral cameras have an improved readout time of $\sim 5$ seconds. The CCD on this camera is a Fairchild CCD 486 with $4096 \times 4096$ pixels.

It is planned that all future telescopes within the LCOGT network (i.e., $0.4 \mathrm{~m}$ and $1.0 \mathrm{~m}$ ) will have similar filter sets. 
Lightcurve for GX 339-4 (Faulkes Telescope South, UVOT on Swift, SAAO $0.75 \mathrm{~m}$ telescope)

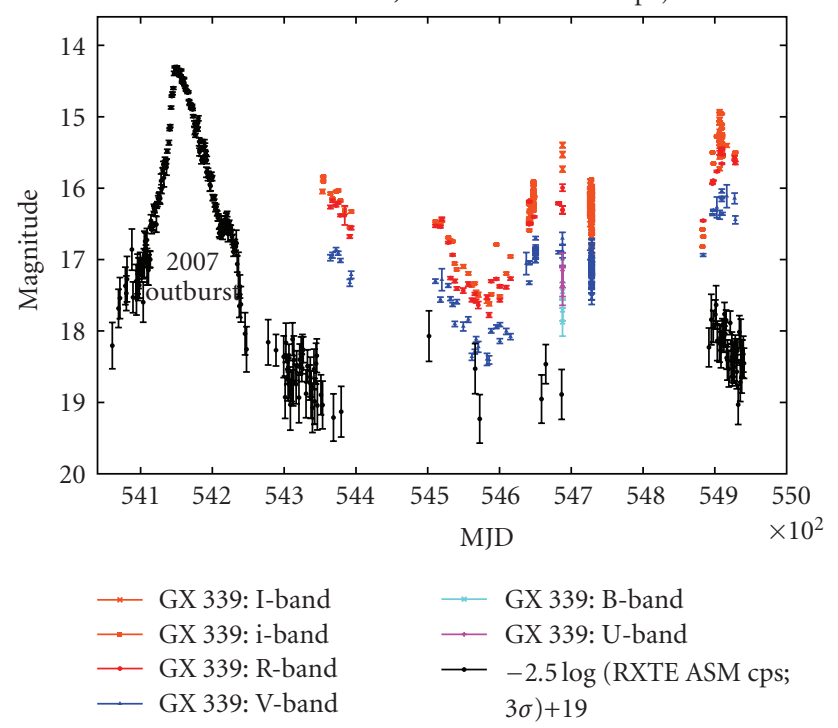

Figure 1: FT (V, R, i'), RXTE ASM, Swift, UVOT (U, B), and SAAO (I) band from 2007 to present.

\section{Scientific Results}

3.1. LMXB Monitoring. The Black Hole X-ray Binary, GX3394. X-Ray binaries (XRBs) consist of a pair of stars orbiting each other; however unlike "normal" binary stars, one of the components is a compact object. This compact object is either a neutron star or a black hole. There are currently $\sim 300$ XRBs in the Milky Way, split into two broad categories based on the mass of the normal component of the system, namely, Low-Mass and High-Mass (LMXBs and HMXBs).

$\mathrm{XRBs}$ possess accretion discs of matter (at temperatures of up to millions of Kelvin), which has been drawn from the "normal" donor star. As well as supernovae, XRBs are also closely linked with phenomena such as Gamma Ray Bursts (GRBs) and as potential sources of gravitational waves.

Initial detections of XRBs are usually made at X-ray wavelengths, a signature of the highly energetic processes involved. They are also observed at other wavelengths, such as the optical regime that the FTs operate within. They can often increase in optical brightness by factors of 1000 or more in a matter of days (known as outbursts) and often display variability on the order of seconds (flickering or flaring).

The low-mass systems (LMXBs) form around 50\% of the overall population-they contain a compact object and a $\mathrm{K}$ or $\mathrm{M}$ type donor star of up to $\sim 1$ solar mass.

Since early 2006, we have been observing a subset of 30 LMXBs with the two FTs [4]. The telescopes are an ideal vehicle with which to monitor these objects, since they allow us the flexibility to change both the frequency and intensity of our observing, subject to changes in the behaviour of our targets. No other survey of LMXBs has covered this many systems so comprehensively or over this time period.

The initial aims of the project are twofold.

(i) We wish to identify outbursts. It is known that many of these systems show increases in brightness at optical wavelengths before being detected in X-rays. Associated with this is the fact that our monitoring is often more sensitive to the initial phase of outburst than typical X-ray monitoring instruments. Identifying these outbursts before other observers allows us to alert the wider astronomical community to initiate multiwavelength follow-up observations (e.g., $[5,6])$.

(ii) Secondly, most XRBs spend a larger fraction of their time as "quiescent" systems rather than in outburst. We wish to constrain this duty cycle and also to compare behaviour in these two states.

On several occasions, we have been able to involve schools and other users in helping us to collect and analyse data. This has led to the schools' contribution being acknowledged in journal papers (e.g., $[7,8]$ ). As well as contributing to professional science, studying XRBs allows students to gain a better understanding of the life cycle of stars, as studied in the science curriculum for schools in the UK.

For systems in outburst, we have been able to collect extensive datasets, incorporating supplementary data at other wavelengths from colleagues, including observatories such as the Very Large Telescope, the Very Large Baseline Array, and the Swift and RXTE X-ray satellites.

One notable target for FT South is the transient black hole binary, GX339-4. This system went into outburst in late 2006 ( MJD 54150), steadily declining in brightness over the following months. The outburst was noted at X-ray [9], optical/infrared [10], and radio wavelengths [11].

Our optical observations (Figure 1) show a continuing decline in V, R, and $i^{\prime}$ bands until $\sim$ MJD 54585, at which time the source began to increase back to its previous brightest level $\left(i^{\prime} \sim 16\right)$ over $\sim 80$ days.

More recent observations ( $\sim$ MJD 54900$)$ show a continued brightening of the source to $i^{\prime} \sim 15$. On several occasions, we have observed rapid flaring in the $i^{\prime}$ and $\mathrm{V}$ bands, with changes of 0.3-0.4 magnitudes in periods of 140 seconds. Our ATel [12] triggered multiwavelength follow-ups with SALT, VLT, Swift and RXTE.

3.2. Observing Massive Stars and Open Clusters: Cyg OB2-B17. Massive stars play an important role in the structure and evolution of galaxies. They are the principal source of heavy elements and UV radiation. They are also the progenitors of energetic phenomena such as X-ray binaries and gamma-ray bursts. However, our understanding of their formation and evolution is still unclear. This is due to their short lifetimes and rapid evolutionary stages, and dust extinction making them difficult to observe during early formation phases.

Some variable massive stars include the following.

(i) OB binaries - the brightest and most massive stellar objects found in galaxies. They are the only direct way to measure the masses and radii of stars, making them the perfect test beds for studying the evolution of massive stars.

(ii) Be Stars-rapidly rotating Be stars which have circumstellar discs at some points during their lives. These are characterised by infrared excess and emission lines. How these discs are formed is still poorly understood. By studying Be stars, we can obtain information on stellar rotation and mixing. 
(iii) Luminous Blue Variables (LBVs)—massive stars close to the upper luminosity limit, causing instabilities in the stellar envelope which results in sporadic mass ejections.

We have been looking for photometric variability of massive stars in the Magellanic Clouds. This survey will be combined with multiepoch spectroscopic data from the VLTFLAMES study (e.g., [13]).

This search has been divided into the following two main parts.

(i) Looking at lightcurves derived from the MACHO microlensing survey [14]. This survey was carried out between June 1992 and December 1999. A 50 inch telescope at Mt. Stromlo, Australia, was used with a dichroic, obtaining simultaneous blue and red observations.

(ii) Photometric monitoring with Faulkes Telescope South. Since October 2007 we have been obtaining offline observations of the clusters NGC2004 (field covered by 5 FTS Merope camera mosaics), and N11 (8 mosaics) in the LMC and NGC346 (1 mosaic) in the SMC. A similar monitoring campaign is now monitoring 30 Doradus in the LMC, where we are obtaining observations using both offline and realtime modes. Much of the real-time data will be collected by schools.

Our aims are to look for the following.

(i) Eclipsing binaries-in order to determine the masses of the components, along with the orbital parameters.

(ii) Variability in Be stars—gaining or losing circumstellar discs.

(iii) Variability due to sporadic mass ejections in Luminous Blue Variables.

(iv) Any transients or new phenomena within the clusters.

The photometric follow-up in the Magellanic clouds is intended to reveal further systems similar to the massive binary, B17 [15].

It is an eclipsing, double-lined spectroscopic binary in the Cyg OB2 association, with two supergiants having preliminary spectral classifications of $\mathrm{O} 7$ and $\mathrm{O}$. The lightcurve reveals a semicontact binary with an orbital period of $4.0217 \pm 0.0004$ days (Figure 2) and indications of mass transfer.

B17 appears to have progenitor masses of $\sim 60$ solar masses, being consistent with the preliminary spectral types of both components as well as the age and stellar population of Cyg OB2. Assuming that the system avoids merger, it is likely to evolve through an extreme B supergiant/LBV phase into a long period Wolf-Rayet + Wolf-Rayet binary configuration, as mass loss via stellar winds increases the orbital separation.

Open clusters are groups of tens to thousands of loosely gravitationally bound stars, formed from the same original molecular cloud. Since the stars within a cluster essentially have the same chemical composition, age, and distance, open clusters are important in the study of stellar evolution.

We have collected observations of many previously unstudied open clusters, as we encourage schools and other

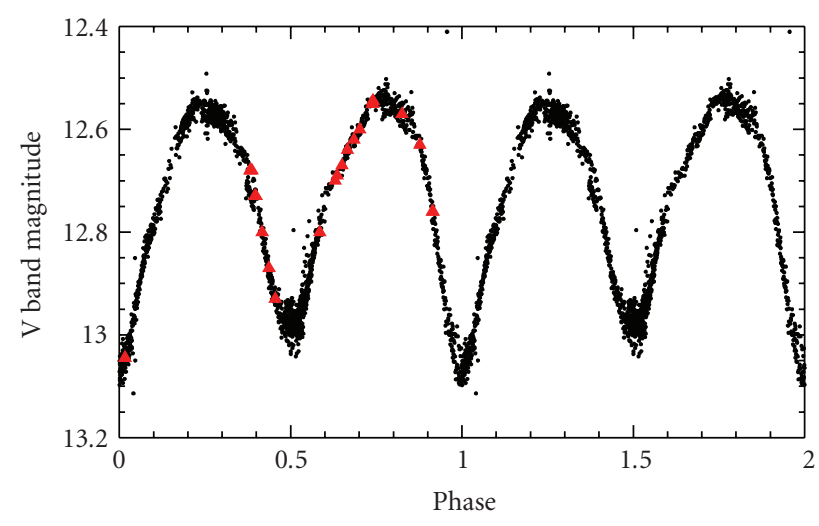

Figure 2: Folded lightcurve of B17 obtained by amateur astronomers. The red triangles show the phases where spectra were obtained [15].

users to analyse. In doing this, they are able to create colourmagnitude diagrams, and search the clusters for $\mathrm{H} \alpha$ bright objects, such as Be stars. Further, they are encouraged to image the clusters themselves to search for variable objects.

3.3. Microlensing Events. LCOGT supports a professional programme to observe microlensing events in the direction of the Galactic Bulge, with the aim of discovering new exoplanets. The observations we provide help to characterise the systems.

With hundreds of events every year, this program relies on a robotic system to prioritise those events which offer the most sensitivity to the presence of planets. In this season, the microlensing team has established a website to provide Faulkes Telescope Project contributors with an updated list of current targets, with advice on making observations of the events using the Real-Time Interface [16]. The images taken by the schools are gathered automatically for processing and combined with data taken by astronomers around the world. This pilot program has received an encouraging response from FT users in its first year.

\section{Future Plans}

LCOGT is expanding its network to include 1-metre and 0.4-metre telescopes running in conjunction with the 2metre FTs [17]. In addition to the imaging capability of the FTs, LCOGT is commissioning a Medium Resolution Echelle Spectrograph and a Low Resolution Spectrograph. LCOGT is designing and building a set of 1-metre telescopes, primarily for use in the LCOGT science program, but with possibly limited time available for educational use. Currently, 12-15 telescopes are planned across 6 sites, each housed in its own custom-made dome. Sites currently being planned are Siding Spring (Australia), Cerro Tololo (Chile), Mauna Loa (Hawaii), Sutherland (South Africa), Tenerife (Canary Islands) and San Pedro Martir (Mexico), with the prototype being despatched to Cerro Tololo in early 2010 .

Currently in production at LCOGT headquarters in Goleta, California, the $0.4 \mathrm{~m}$ telescopes are based around 
an off-the-shelf Meade telescope with a custom equatorial mount, and high specification CCD cameras. It is anticipated that these telescopes will mainly be available for the education community and other nonprofessional astronomers to use. Despite their relatively small size, they will be installed at dark sites in pairs or sets of fours, allowing high-quality imaging with wider fields of view $\left(\sim 30^{\prime} \times 20^{\prime}\right)$ than those of the larger aperture telescopes. They will feature the SBIG STL-6303 with a Kodak KAF 6303E CCD with $2048 \times 3072$ pixels.

Two of these telescopes are now located inside the enclosures of each FT. Two to four telescopes will be installed at the other $1.0 \mathrm{~m}$ sites. When fully commissioned, they will become available to the user community. With the exception of those $0.4 \mathrm{~m}$ telescopes housed within the FTs, the remainder of the $0.4 \mathrm{~m}$ network will be placed within their own Aqawan enclosures, each capable of housing two telescopes [18].

\section{Acknowledgments}

The first and the fourth authors acknowledge the financial support from the Dill Faulkes Educational Trust and The Open University. The fifth author acknowledges the support from a Netherlands Organization for Scientific Research (NWO) Veni Fellowship. ASM/RXTE data are provided by the ASM/RXTE team.

\section{References}

[1] F. Lewis and P. Roche, "Not just pretty pictures," in Astronomy: Networked Astronomy and the New Media, R. J. Simpson and D. Ward-Thompson, Eds., 2009.

[2] Y. Tsapras, R. Street, K. Home, et al., "RoboNet-II: follow-up obervations of microlensing events with a robotic network of telescopes," Astronomische Nachrichten, vol. 330, no. 1, pp. 411, 2009.

[3] http://lcogt.net/en/network/camera/merope-llc-merope.

[4] F. Lewis, D. M. Russell, R. P. Fender, P. Roche, and J. S. Clark, "Continued monitoring of LMXBs with the Faulkes Telescopes," in Proceedings of the 7th Microquasar Workshop: Microquasars and Beyond, 2008.

[5] F. Lewis, M. Linares, D. M. Russell, R. Wijnands, and P. Roche, "Renewed optical and X-ray activity in IGR J00291+5934," ATel, no. 1726, 2009.

[6] D. M. Russell and F. Lewis, "Optical and hard X-ray detections of an outburst from Aquila X-1," ATel, no. 1970, 2009.

[7] P. Elebert, M. T. Reynolds, P. J. Callanan, et al., "Optical spectroscopy and photometry of SAX J1808.4-3658 in outburst," Monthly Notices of the Royal Astronomical Society, vol. 395, no. 2, pp. 884-894, 2009.

[8] M. R. Burleigh, T. R. Marsh, B. T. Gänsicke, et al., "The nature of the close magnetic white dwarf + probable brown dwarf binary SDSS J121209.31+013627.7," Monthly Notices of the Royal Astronomical Society, vol. 373, no. 4, pp. 1416-1422, 2006.

[9] H. A. Krimm, L. Barbier, S. D. Barthelmy, et al., "Swift-BAT detects a bright hard X-ray outburst from GX339-4," ATel, no. 968, 2006.

[10] M. Buxton and C. Bailyn, "Latest optical and infrared observations of GX 339-4,” ATel, no. 1027, 2007.
[11] S. Corbel, T. Tzioumis, C. Brocksopp, and R. Fender, "ATCA radio observations of GX339-4," ATel, no. 1007, 2007.

[12] D. M. Russell, D. Altamirano, F. Lewis, P. Roche, C. B. Markwardt, and R. P. Fender, "Unusual optical and X-ray flaring activity in GX339-4," ATel, no. 1586, 2008.

[13] C. J. Evans, D. J. Lennon, S. J. Smartt, and C. Trundle, "The VLT-FLAMES survey of massive stars: observations centered on the Magellanic Cloud clusters NGC 330, NGC 346, NGC 2004, and the N11 region," Astronomy and Astrophysics, vol. 456, no. 2, pp. 623-638, 2006.

[14] C. Alcock, R. A. Allsman, D. R. Alves, et al., "Calibration of the MACHO photometry database," The Publications of the Astronomical Society of the Pacific, vol. 111, no. 766, pp. 15391558, 1999.

[15] V. E. Stroud, S. Clark, I. Negueruela, P. D. Roche, A. J. Norton, and F. Vilardell, "Discovery of the eclipsing of supergiant binary, Cyg OB2-B17," submitted to Astronomy \& Astrophysics, http://arxiv.org/abs/1002.0707.

[16] http://microlensing.lcogt.net.

[17] M. G. Hidas, E. Hawkins, Z. Walker, T. M. Brown, and W. E. Rosing, "Las Cumbres Observatory Global Telescope: a homogeneous telescope network," Astronomische Nachrichten, vol. 329, no. 3, pp. 269-270, 2008.

[18] http://lcogt.net/en/blog/egomez/how-keep-telescope-dry. 

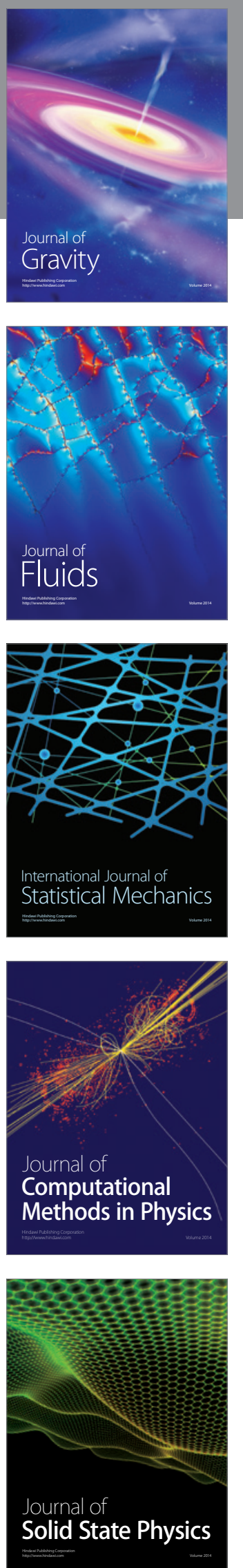

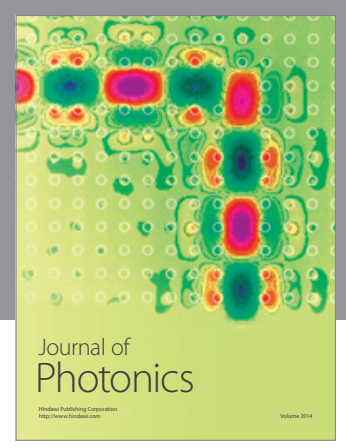

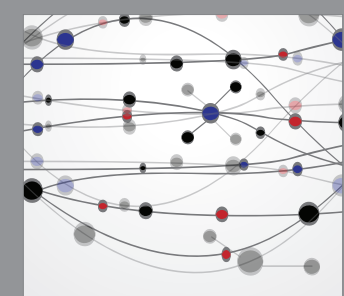

The Scientific World Journal
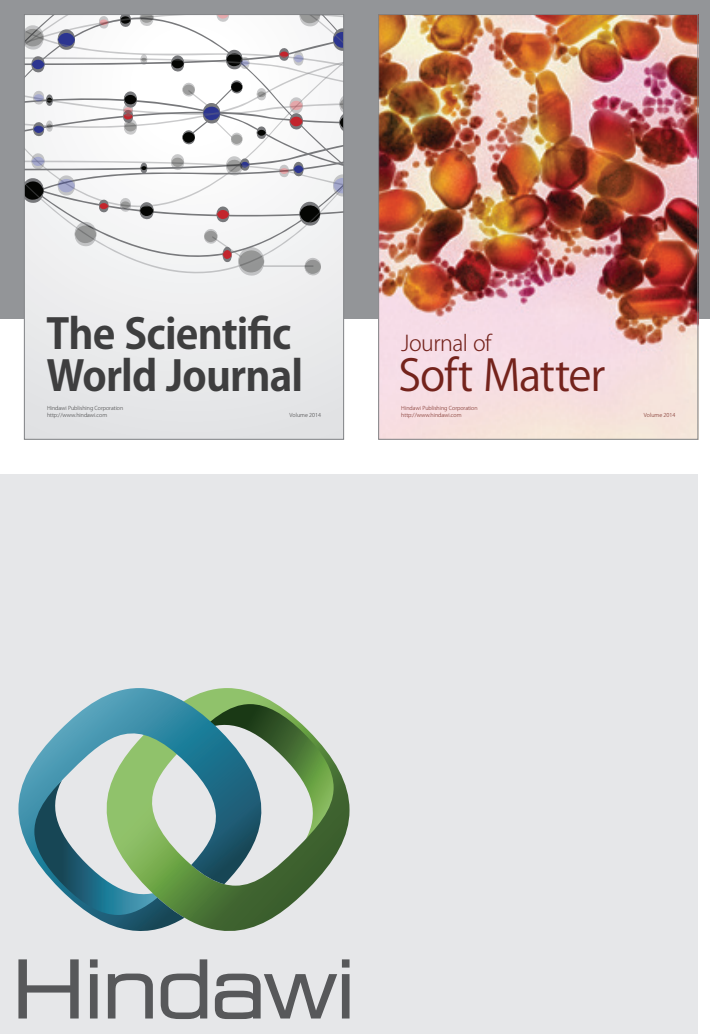

Submit your manuscripts at

http://www.hindawi.com
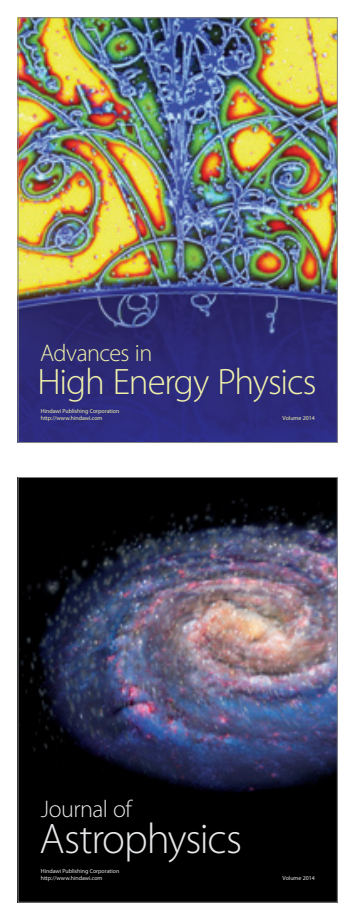
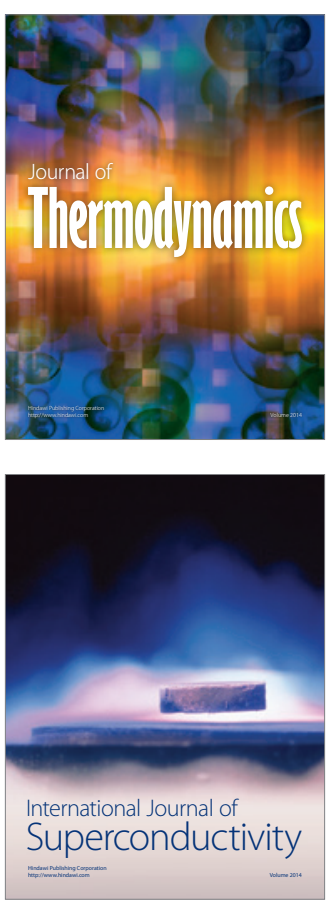
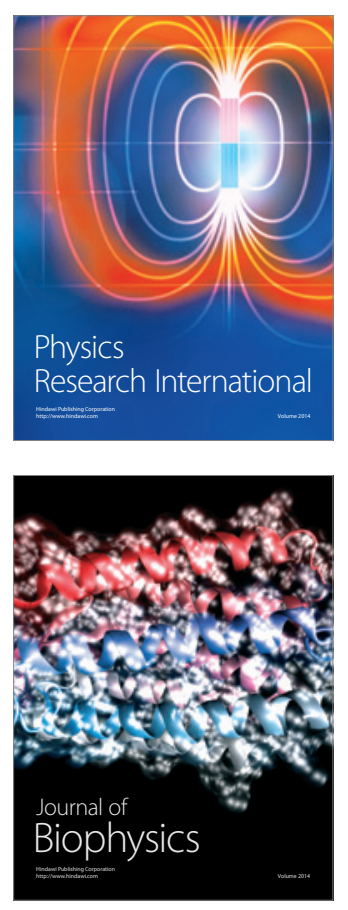
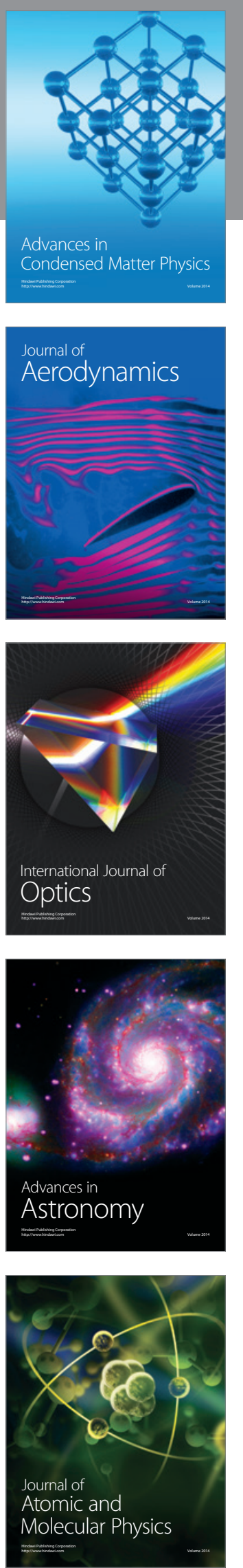